\title{
Research on stabilization control method for humanoid robot
}

\author{
Jianming LOU $^{1}$ \\ 1.School of Electronic and Information Engineering, \\ Ningbo University of Technology, \\ Ningbo 315016, China \\ ljm842@163.com
}

Qiubo ZHONG ${ }^{1,2}$ Xiaohui ZHANG ${ }^{1}$

\begin{abstract}
For the characteristics of humanoid robot in stability control, a method called second-order cone planning is proposed for controlling the stability of robot walking. The stability control of humanoid robot is configured to convert the second order cone to be suitable for solving the problem by derivating a variety of stability constraints of robot during the walking movement. The effectiveness of the proposed method is validated through simulation and experiments
\end{abstract}

Keywords — humanoid robot; second order cone; stability constraint

\section{INTRODUCTION}

Recently, research of humanoid robot gets more and more attention in researchers at home and abroad. In the research of humanoid robot, the stability study of robot is one of the key technologies in humanoid robot motion. In this aspect, numerous researchers do a lot related researches. At humanoid robot stability control, ZMP is the most important factor to realize humanoid robot stabilize motion. In 2003[1], Kajita controls motion of robot by compensation ZMP, and gets good results. In 2007[2], some basic factors required by balancing humanoid robot put forward. These factors including ZMP (Zero moment point), center of gravity Jacoby matrix and so on, control the motion of humanoid robot by embedded in the action adding center of gravity matrix. There also use fuzzy controller based on ZMP error. First give centroid position increment of robot, and then control motion by quadratic programming method and centroid Jacoby matrix solve correction value for robot each joint angles method. Humanoid robot often requires ZMP, center of gravity and other stability criterion and the range of moment, friction influence under the various environment and any constraints at same time, because of robot is always in a multi-degree of freedom joint control system of complex environments. Therefore there needed a way to control multiple performance tuning index and constraint at same time.

SOCP (Second-Order Cone Programming) [3] is an optimal design method which can take into account a number of indicators. For norm criteria, including single-norm and mixed-norm can be achieved by SOCP[4], not only that, robust filter can be achieved. The main idea of SOCP is that objective function in optimal problem can translate into constraint function by introducing variables, then through suitable transform, new objective variable and original variables to be optimized can be combined into new optimized variable, and the original SOCP constraint can transform into SOCP constraint about new optimized variable, finally, as long as objective function and constraint function in the optimization problem can be expressed in the form of SOCP, the optimization problem can be solved using the SOCP. SOCP has been used in filter design optimization problems and beam-former optimization problem widely, and get a good practical effect. Y. Q. Bai, M. EL GHami and C. Roos[5,6] discuss using kernel function to solve dual interior point algorithm of SOCP and linear programming

\author{
2. State Key Laboratory Of Robotics and System, \\ Harbin Institute of Technology, \\ Harbin 150001, China \\ zhongqiubo@gmail.com
}

problems. Basing on advantages of SOCP control, it has a great practical significance for control technology and design optimization of the walking motion in the humanoid robot.

\section{SOCP CONTROL THEORY}

SOCP is a subset of the convex programming. It is under the condition of satisfy a set of SOCP constraints and linear equality constraints so that a linear function is minimized, it is expressed as[7]:

$$
\begin{aligned}
& \underset{\boldsymbol{y}}{\min } \boldsymbol{b}^{T} \boldsymbol{y} \\
& \text { Subject to }\left\|\boldsymbol{A}_{i} \boldsymbol{y}+\boldsymbol{b}_{i}\right\| \leq \boldsymbol{c}_{i}^{T} \boldsymbol{y}+d_{i} \\
& \boldsymbol{i}=1,2, \cdots, \boldsymbol{I} \\
& \boldsymbol{F} \boldsymbol{y}=\boldsymbol{g}
\end{aligned}
$$

Where $\boldsymbol{b} \in C^{\alpha+1}$ means parameter of the solving problem, $\boldsymbol{y} \in C^{\alpha+1}$ means variable of the optimization, $\boldsymbol{A}_{i} \in C^{\left(\alpha_{i}-1\right) \times \alpha}, \boldsymbol{b}_{i} \in C^{\left(\alpha_{i}-1\right) \times 1}, \boldsymbol{c}_{i} \in C^{\alpha \times 1}, \boldsymbol{c}_{i}^{T} \boldsymbol{y} \in R$, $\boldsymbol{d}_{i} \in R, \boldsymbol{F} \in C^{g \times \alpha}, \boldsymbol{g} \in C^{g \times 1},\|$.$\| means Euclid norm,$ such as $\|u\|=\left(u^{T} u\right)^{1 / 2},(\cdot)^{T}$ means transposition, $C$ means plural sets, $R$ means real sets. (1b):

Each constraint can be expressed as second-order cone in

$$
\left[\begin{array}{c}
\boldsymbol{c}_{i}^{T} \\
\boldsymbol{A}_{i}
\end{array}\right] \boldsymbol{y}+\left[\begin{array}{l}
d_{i} \\
\boldsymbol{b}_{i}
\end{array}\right] \in S O C_{i}^{\alpha_{i}}
$$

$S O C_{i}^{\alpha_{i}}$ is the second-order cone of space of $C^{\alpha_{i}}$, and defined as:

$$
\operatorname{SOC}_{i}^{\alpha_{i}} \stackrel{\Delta}{=}\left\{\left[\begin{array}{l}
t \\
\boldsymbol{x}
\end{array}\right] \mid t \in R, \boldsymbol{x} \in C^{\left(\alpha_{i}-1\right) \times 1},\|\boldsymbol{x}\| \leq t\right\}
$$

The Geometrical meaning of SOCP optimization is seeking the most advantage which meeting the objective function minimization in 3D second-order cone.

Equality constraints in (1c) can be expressed as zero-cone:

Zero-cone can be defined as:

$$
\boldsymbol{g}-\boldsymbol{F} \boldsymbol{y} \in\{\boldsymbol{0}\}^{g}
$$

$$
\{\boldsymbol{0}\}^{g} \stackrel{\Delta}{=}\left\{\boldsymbol{x} \mid \boldsymbol{x} \in C^{\boldsymbol{g} \times 1}, \boldsymbol{x}=\mathbf{0}\right\},
$$

As shown in (1), when $\alpha_{i}=1, i=1, \ldots, N$, linear programming(linear inequality constraints)and convex quadratic programming is a special case of SOCP.

Formula (1a) is the objective function, which is a set of 
D-value of state variables and object variables, when the D-value tends to infinity, can get the optimal a set of state variables, and all of state variables from a conical which combining with formula (1b) and (1c), formula (1b) means shapes in the cone except conical bottom, and formula (1c) means conical bottom. Iterating any set of control variable meeting formula (1b) and (1c), the corresponding state variable using formula (1a) for comparison, after reaching iterative times or meeting fixed solving accuracy, it stop. Then, the state from control variable is an optimal state.

\section{CONSTRUCT BALANCE CONSTRAINT FOR WALKING MOTION IN HUMANOID ROBOT}

Because of center of gravity in humanoid robot is high, area for feet supporting is small, and these features make humanoid robot become an unstable structure during motion. Using the way of restricting humanoid robot in ZMP, friction and kinetic energy during walking, control stability during movement effectively.

\section{A. ZMP constraint}

According to the definition of ZMP[8], when ZMP in the polygon, range of the ZMP can be expressed as:

$$
p_{z m p} \in\left\{\sum_{i=1}^{N} \alpha_{i} p_{i} \mid p_{i} \in S(i=1,2, \cdots, N)\right\}
$$

Among them, $\alpha_{i}=f_{i z} / \sum_{i=1}^{N} f_{i z}$ is the vertical component ratio of force from ground. $p_{i} \in S(i=1,2, \cdots N)$ is the discrete point in the support region. If given matrix $A_{s}$ and vector $b_{s}$, and can construct inequality which has same meaning with formula (2):

$$
A_{s} p_{z m p}+b_{s} \leq 0
$$

Usually, in the condition of meeting (2), given a desired ZMP locus $p_{z m p}^{d}$ as input, there is an error $\varepsilon_{z m p}$ between actual ZMP and desired ZMP meeting the following inequality relations:

$$
\left\|p_{z m p}-p_{z m p}^{d}\right\| \leq \varepsilon_{z m p}
$$

\section{B. $\quad$ Friction constraints}

In order to prevent the footsteps slide of robot, we should consider both of strong ground tangent and normal movement. In the case of robot on the ground with one foot, basic friction constraints can expressed as following:

$$
\begin{gathered}
\left\|P_{x y} F_{o}\right\| \leq \mu F_{1} \\
\left\|P_{m z} F_{o}-p_{z m p} \times P_{x y} F_{o}\right\| \leq \mu_{r} F_{2} \\
F_{1}+F_{2} \leq P_{z} F_{o}
\end{gathered}
$$

$P_{x y}, P_{m z}$ and $P_{z}$ is force vector of xy plane, and projection matrix that force tangent with $\mathrm{z}$ coordinate. $\mu$ is coefficient of friction, $\mu_{r}$ is twist coefficient of friction when it move normally, $F_{0}, F_{1}$ and $F_{2}$ is frictional resistance from robot during forward movement, tangential movement and normal movement respectively. We need determine sum of compelling force less than $P_{z} F_{o}$ for static pace.

\section{Kinetic constraints}

If rotation angle response of rotating joint of humanoid robot is $\boldsymbol{q}=[\dot{q}(1), \dot{q}(2), \cdots, \dot{q}(L)]^{T}$, at a sport moment for humanoid robot, it can be expressed as:

$$
T(t)=\sum_{l=1}^{L} q(l) p(l)=\boldsymbol{p}^{T}(l) \boldsymbol{q}
$$

Among $\boldsymbol{p}(t)=\dot{\boldsymbol{q}}^{T} \boldsymbol{J}(i j), \boldsymbol{J}$ means vector matrix of inertia.

If temporal sample points meeting $f_{k} \in F(k=1,2, \cdots, K)$, these sample points have uniform or non-uniform interval. Solving problem about dynamic equilibrium of humanoid robot is making the following error weighted norm minimum

$$
\left\{\sum_{k=1}^{K} \lambda_{k}\left|T_{d}\left(t_{k}\right)-T\left(t_{k}\right)\right|^{p}\right\}^{1 / p}
$$

Where, $T_{d}\left(t_{k}\right)$ is expected response of optimized design when time is $t_{k}$, is non-negative weighting factor, for adjusting fitted closeness for different parameters. Typically, range of error take $L_{1}, L_{2}$ or norm $L_{\infty}$ that $p=1,2, o r, \infty$. The optimal control problem of kinetic energy under the rule of this three norm types expressed as following:

$$
\begin{gathered}
\min _{\boldsymbol{q}} \sum_{k=1}^{K}\left(\lambda_{k}\left|T_{d}\left(t_{k}\right)-\boldsymbol{p}^{T}\left(t_{k}\right) \boldsymbol{q}\right|\right) \\
\min _{\boldsymbol{q}} \sum_{k=1}^{K} \lambda_{k} \mid T_{d} \boldsymbol{t}_{k}-\boldsymbol{p}^{T} \quad\left(\left.\boldsymbol{q}\right|^{2}\right. \\
\min _{\boldsymbol{q}} \max _{k}\left(\lambda_{k}\left|T_{d}\left(t_{k}\right)-\boldsymbol{p}^{T}\left(t_{k}\right) \boldsymbol{q}\right|\right)
\end{gathered}
$$

\section{STRUCTURE AND SOLUTION OF SOCP}

Take $L_{2}$ norm standard from formula (11) for example, we can translate optimization problems into SOCP problem for solving.

Introducing a set of non-negative variable $\varepsilon_{k}(k=1,2, \cdots, K)$, formula (11) can be expressed like this:

$$
\begin{gathered}
\min _{\boldsymbol{q}} \sum_{k=1}^{K}\left(\lambda_{k} \varepsilon_{k}\right) \quad \text { subject } \\
\left|T_{d}\left(t_{k}\right)-\boldsymbol{p}^{T}\left(t_{k}\right) \boldsymbol{q}\right|^{2} \leq \varepsilon_{k}, \quad k=1,2, \cdots, K \\
\text { Constraint condition is formula (14)-(18): } \\
A_{s} p_{z m p}+b_{s} \leq 0 \\
\left\|p_{z m p}-p_{z m p}^{d}\right\| \leq \varepsilon_{z m p} \\
\left\|P_{x y} F_{o}\right\| \leq \mu F_{1} \\
\left\|P_{m z} F_{o}-p_{z m p} \times P_{x y} F_{o}\right\| \leq \mu_{r} F_{2}
\end{gathered}
$$




$$
F_{1}+F_{2} \leq P_{z} F_{o}
$$

Quadratic inequality constraint from formula (13), have

$$
\begin{aligned}
& \left|T_{d}\left(t_{k}\right)-\boldsymbol{p}^{T}\left(t_{k}\right) \boldsymbol{q}\right|^{2} \leq \varepsilon_{k} \\
& \Leftrightarrow\left|2 T_{d}\left(t_{k}\right)-2 \boldsymbol{p}^{T}\left(t_{k}\right) \boldsymbol{q}\right|^{2}+1+\varepsilon_{k}^{2}-2 \varepsilon_{k} \leq 1+\varepsilon_{k}^{2}+2 \varepsilon_{k} \\
& \Leftrightarrow\left\|\begin{array}{c}
2 T_{d}\left(t_{k}\right)-2 \boldsymbol{p}^{T}\left(t_{k}\right) \boldsymbol{q} \\
\varepsilon_{k}-1
\end{array}\right\|^{2} \leq\left(\varepsilon_{k}+1\right)^{2} \\
& \Leftrightarrow\left\|\begin{array}{c}
2 T_{d}\left(t_{k}\right)-2 \boldsymbol{p}^{T}\left(t_{k}\right) \boldsymbol{q} \\
\varepsilon_{k}-1
\end{array}\right\| \leq \varepsilon_{k}+1
\end{aligned}
$$

Defining

$$
\boldsymbol{y}=\left[\varepsilon_{1}, \varepsilon_{2}, \cdots, \varepsilon_{K}, \boldsymbol{q}^{T}\right]^{T}
$$

and

$\boldsymbol{b}=\left[\lambda_{1}, \lambda_{2}, \cdots, \lambda_{K}, \mathbf{0}_{1 \times L}\right]^{T}$ make $\boldsymbol{b}^{T} \boldsymbol{y}=\sum_{k=1}^{K}\left(\lambda_{k} \varepsilon_{k}\right)$,

$\mathbf{0}_{1 \times L}$ means $1 \times L$ zero dimensional vector, formula $(13$

become $\min _{\boldsymbol{y}} \boldsymbol{b}^{T} \boldsymbol{y}$ subject to:

$$
\begin{array}{r}
\left\|\left[\begin{array}{c}
2 T_{d}\left(t_{k}\right) \\
-1
\end{array}\right]-\left[\begin{array}{cc}
\mathbf{0}_{1 \times K} & 2 \boldsymbol{p}^{T}\left(t_{k}\right) \\
-\boldsymbol{m}^{T}(k) & \mathbf{0}_{1 \times L}
\end{array}\right] \boldsymbol{y}\right\| \\
, k=1+\left[\begin{array}{ll}
\boldsymbol{m}^{T}(k) & \mathbf{0}_{1 \times L}
\end{array}\right] \boldsymbol{y} \\
, k=1, \cdots, K
\end{array}
$$

Among them, $\boldsymbol{m}(k)=\left[m_{1}, m_{2}, \cdots, m_{n}, \cdots, m_{K}\right]^{T}$, $m_{i}=\left\{\begin{array}{l}0, n \neq k, \\ 1, n=k .\end{array}\right.$

For the formula (20), we can use interior point method from penalty function[7] for solving and more details can be seen there.

\section{SIMULATION AND EXPERIMENT}

Simulation use Matlab own toolkit of SOCP for optimal solution of stability control of humanoid robot. It's assumed that torque of humanoid robot can keep enough dynamic balance, and assume friction coefficient when robot walk on the ground is $\mu=0.4$, friction coefficient of joint twisting is $\mu_{r}=0.45$.

We compare LMS adaptive method with SOCP design method, achieving control of dynamic balance for humanoid robot at any time nodes by Matlab simulation, and compare results of iterative error and results of displacement error with PSO that have good results.

It choose the test time is 1s, researching humanoid robot when waling during this time can satisfied requirement of the stability of the instantaneous walking, choose a stepping when time node is $0.1 \mathrm{~s}$, and assume velocity of humanoid robot is $v=0.3 \mathrm{~m} / \mathrm{s}$, quality of humanoid robot is $\mathrm{m}$, then expected value of kinetic energy from robot is $0.045 \mathrm{~kg} . J$, the simulation result shown in figure 1,2. Figure 1 is kinetic simulation of humanoid robot motion, and figure 2 is the result of comparing walking optimized design of two methods of humanoid robot in delay respects.

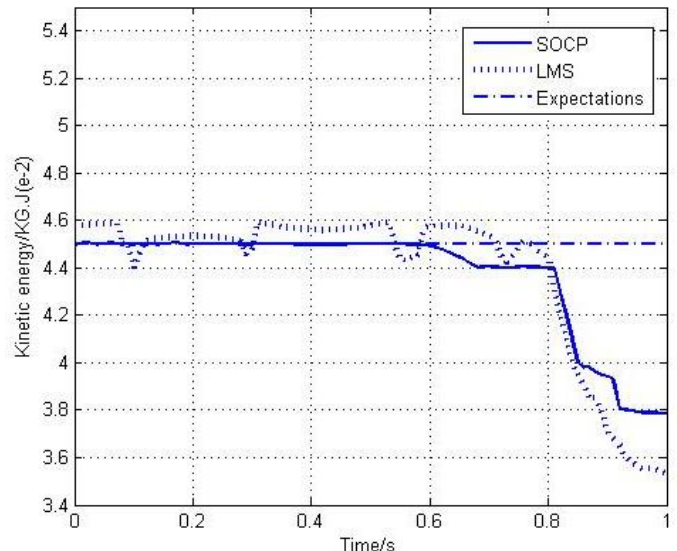

Figure 1 kinetic change with time

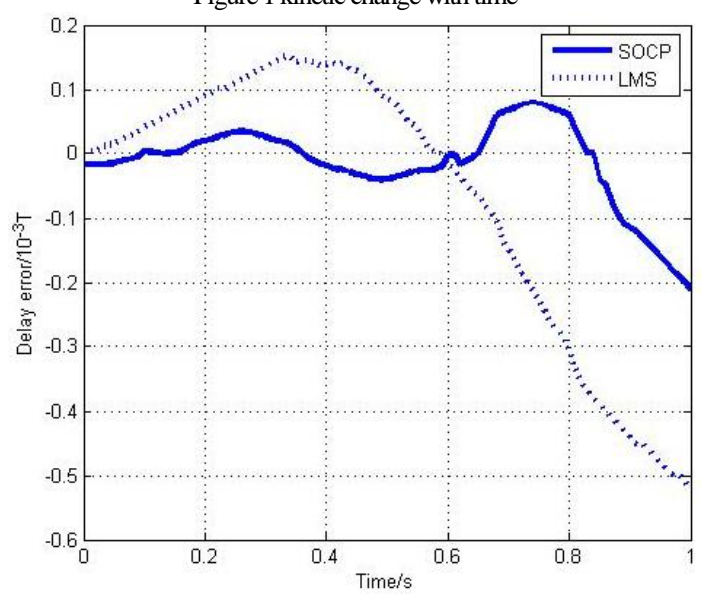

Figure 2 Delay error

It compares results of iterative error and results of displacement error with PSO respectively for reflecting characteristic when control the stability of robot in optimization.

Shown in figure 2 is comparing SOCP with PSO in result of iterative error, choosing dimension is 28-demensional particle swarm, numbers of particle are 30 , both study factor $c_{1}, c_{2}$ are 2 , inertia weight $\mathrm{w}$ is 0.7 , iterative steps are 700 .

Shown in figure 3 is comparing SOCP with PSO in result of displacement error, specify humanoid robot walking along a straight line which be marked, test distance is $6 \mathrm{~m}$, optimize robot gait by SOCP and PSO respectively, and test error of robot deviating from marked route.

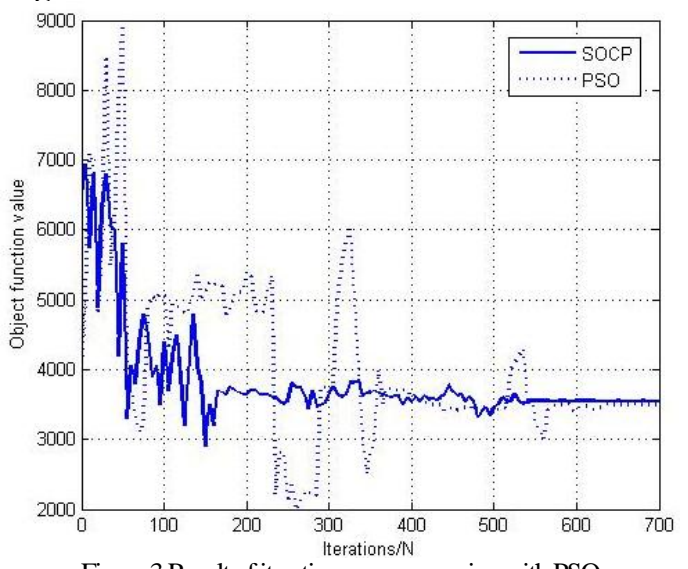

Figure 3 Result of iterative error comparing with PSO 


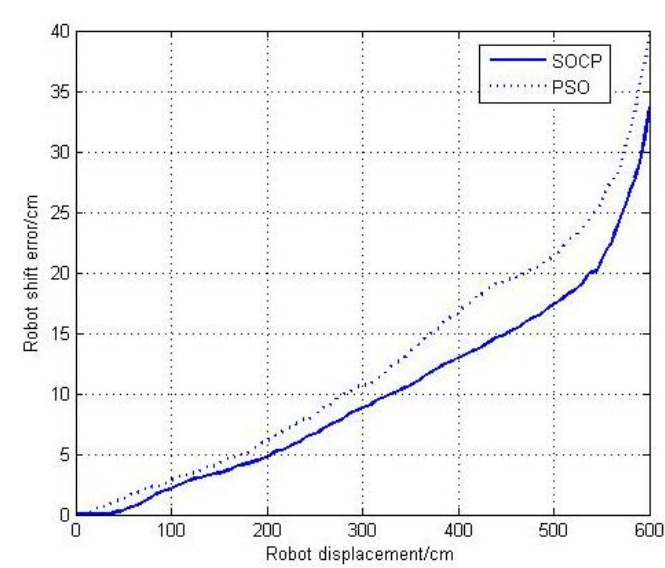

Figure 4 Result of displacement error comparing with PSO

As shown in figure 3, convergence of SOCP is quicker than PSO when it started in the result of iterative error, and tends to optimal values when iteration is about 280 , and the shock of PSO is strong, it tends to steady when iteration is about 580 .

During the humanoid robot walking $6 \mathrm{~m}$ along the marked straight line, it will deviate the marked route inevitably, because of not-smooth surface and other constraints of objective factors and robot don't have visual navigation, shown in figure 4, SOCP is better than PSO in the result of displacement error.

\section{CONCLUSION}

Control system of humanoid robot walking is a multi-DOF and nonlinear optimized control system, using SOCP to translate optimal control problem of walking stability into SOCP optimization problem for the complexity of its control, and simulate controlling walking robot by simulation software, compare with various common methods and analysis at same time. According to the simulation, this way is useful to optimization problem in controlling stability of humanoid robot with complex constraints, specifically when reduce the optimization parameters, it can achieve optimization online.

Experimental results show that, this method can control walking of humanoid robot effectively and be sure its stability of walking.

\section{ACKNOWLEDGEMENT}

This material is based upon work funded by Natural Science Foundation of China under Grant No.61203360, Zhejiang Provincial Natural Science Foundation of China under Grant No.LQ12F03001, LQ12D01001, LY12F01002, Ningbo City Natural Science Foundation of China under Grant No.2012A610009, 2012A610043, State Key Laboratory of Robotics and System (HIT)Foundation of China under Grant No.SKLRS-2012-MS-06, China Postdoctoral Science Foundation under Grant No.2013M531022

\section{REFERENCES}

[1] Kajita, S.; Kanehiro, F.; Kaneko, K.; Fujiwara, K.; Harada, K.; Yokoi, K. \& Hirukawa, $\mathrm{H}$. Biped walking pattern generation by using preview control of zero-moment point, Proc. of IEEE Int. Conf. on Robotics and Automation, 2003: $1620-1626$

[2] Choi, Y.; Kim, D.; Oh, Y. \& You, B-J. Walking Control of Humanoid Robot based on the Kinematic Resolution of CoM Jacobian with Embedded Motion, IEEE Trans. on Robotics, Vol 23, No. 6, 2012:1285-1293.

[3] Lobo M, Vandenberghe L, Boyed S et al. Applications of second-order cone programming. Linear Algebra Applicat, 1998, 284(1 3): 193-228.

[4] Yan S F, Ma Y L. A unified framework for designing FIR filters with arbitrary magnitude and phase reponse. Digital Signal Processing, 2004,
14(6): 510-522.

[5] Bai Y Q. Qang G Q, Roos C. Anew primal-dual interior-point algorithm for second-order cone optimization [EB/OL]. http://www.optinization-online org/DB-FILE/2004/11/1001.pdf. November 14, 2004.

[6] Bai Y Q. Ghamiand M EI, Roos C. A comparative study of kernel functions for primal-dual interior-point algorithms in linear optimization [J]. SMA Journalon Optinization, 2004 15(1): 101-128.

[7] Lobo M S, Vandenverghe L, Boyd S, et al. Applications of second-order cone programming[J]. Linear Algebra Application, 1998:193-228.

[8] Vukobratovic M, Stepanenko J. On the stability of anthropomorphic systems[J]. Mathematical Biosciences, 1972, 15:1-37. 